\title{
Plaque Longitudinal Heterogeneity in Morphology, Property, and Mechanobiology
}

\author{
Phani Kumari Paritala ${ }^{a} b \quad$ Tejasri Yarlagadda ${ }^{b}$ Jessica Benitez Mendieta ${ }^{a, b}$ \\ Jiaqiu Wang ${ }^{a, b}$ Tim McGahan $^{c}$ Thomas Lloyd $^{d}$ Prasad K.D.V. Yarlagadda ${ }^{a, b}$ \\ Zhiyong Li ${ }^{a}$, b, e \\ aSchool of Mechanical, Medical and Process Engineering, Queensland University of Technology, Brisbane, QLD, \\ Australia; ' Institute of Health Biomedical Innovation (IHBI), Queensland University of Technology, Brisbane, QLD, \\ Australia; 'Department of Vascular Surgery, Princess Alexandra Hospital, Brisbane, QLD, Australia; ${ }^{\mathrm{d} D e p a r t m e n t}$

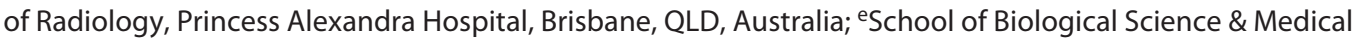 \\ Engineering, Southeast University, Nanjing, China
}

\section{Keywords}

Carotid plaque $\cdot$ Heterogeneity $\cdot$ Immunostaining $\cdot$

Computational fluid dynamics $\cdot$ Stress analysis

\section{Abstract}

Background and Purpose: The hemodynamic environment of an atherosclerotic plaque varies along the longitudinal direction. Investigating the changes in plaque morphology and its biomechanical environment along the longitudinal direction and their correlations will enhance our understanding of plaque progression and arterial remodeling. Methods: Six male patients with carotid stenosis $>70 \%$ were recruited. Multisequence high-resolution MRI was performed at the carotid bifurcation. Carotid endarterectomy was performed following MRI, and the plaque tissue was collected for histological and mechanical testing. Patient-specific biomechanical modeling and simulations were conducted to calculate the mechanical stresses (wall shear stress [WSS] and von Mises stress [VMS]). Changes in plaque crosssectional morphology, WSS, and VMS as well as their correlations were evaluated. Results: Positive correlations were found between $\%$ stenosis and $\%$ inflammation (MA) $(p=$

karger@karger.com

(c) 2021 S. Karger AG, Basel

www.karger.com/ced

Karger"
$0.019), \%$ lipid area and \% MA ( $p=0.026)$, and \% calcification area and VMS $(p=0.007)$. Negative correlations were found between VMS and \% stenosis ( $p=0.028)$ and VMS and average WSS $(p=0.034)$. Moreover, the peak stresses and neovessels were found to be in the shoulder regions. High-stress concentrations were found in the interface regions of the calcification and surrounding tissue, thereby increasing plaque vulnerability. Conclusions: Correlations between the morphology and stresses suggest that arterial remodeling is a dynamic interaction between mechanical environment and plaque progression resulting in plaque heterogeneity. Our finding indicates that plaque heterogeneity is associated with plaque progression and can be combined with mechanical stresses for identifying high-risk plaques.

(c) 2021 S. Karger AG, Basel

\section{Introduction}

Atherosclerosis, a significant cause for most of the cardiovascular events, is a chronic inflammatory disease associated with the accumulation of lipids activated by complex interactions between systemic, hemodynamic, 
and biological factors [1]. Consequently, atherosclerotic plaque rupture is the leading cause of myocardial infarction and an important cause of ischemic stroke that result in mortality and long-term morbidity [2]. Although with increasing use of lipid-lowering drugs fewer strokes are due to atherosclerosis and more are from small vessel diseases [3], it is important to understand the mechanobiology and heterogeneity of the atherosclerotic plaque. The mechanical stresses induced in the arteries due to the hemodynamic blood flow play an essential role in plaque progression and remodeling. Histological staining [4] and patient-specific biomechanical simulations [5-7] have been used to identify the morphological features and geometries susceptible to rupture. However, there is still a very limited understanding of how biomechanics impact plaque progression and what leads to a vulnerable plaque formation.

Plaque is a 3-dimensional structure. Its mechanical properties also vary significantly along the longitudinal direction. Hence, to better access plaque vulnerability, it is vital to enhance the understanding of the plaque heterogeneity in the longitudinal direction. Furthermore, it is required to determine the tissue-specific mechanical properties in order to evaluate the correlation between plaque morphology and stresses. Although different techniques have been used for the mechanical characterization of plaque tissue $[8,9]$, the nanoindentation technique is more applicable for extracting tissue-specific properties [10]. In addition, stresses are affected by the interactions between blood flow, vessel geometry, and plaque morphology. Therefore, this study aims to understand the longitudinal heterogeneity of the plaque and assess the correlation between stresses and morphology.

\section{Materials and Methods}

\section{Data Collection}

Six male patients with carotid stenosis $>70 \%$ were recruited from Princess Alexandra Hospital (PAH). Patient demographics can be found in online suppl. Table 1 (for all online suppl. material, see www.karger.com/doi/10.1159/000515690). Multisequence high-resolution MRI was performed at the carotid bifurcation using a multicontrast imaging study in a $3 \mathrm{~T}$ whole body system and with a 64-channel head/neck coil. Carotid endarterectomy was performed following MRI. Carotid plaque samples were collected for histological analysis and mechanical testing.

Plaque Sample Preparation and Sectioning

After excision, plaque samples were cut into 3 to 4-mm-thick segments, embedded in the optimal cutting temperature compound (Tissue-Tek OCT; TED Pella Inc., Redding, CA, USA), and snap-frozen using liquid nitrogen before storing at $-20^{\circ} \mathrm{C}$ within
$2 \mathrm{~h}$ of excision. Each segment was used to section $8-\mu \mathrm{m}$ (for histology) and 30- $\mu \mathrm{m}$ (for mechanical testing) thick slices using a cryostat (Leica CM1850) [11].

\section{Histological Analysis and Immunostaining}

Leica XL ST5010 Autostainer (Leica Biosystems, Buffalo Grove, IL, USA) was used to perform standard hematoxylin and eosin staining to define the basic morphology. Oil Red $\mathrm{O}$ was used to define the lipids, and Masson's trichrome was used to define the fibrous tissue and hemorrhage. Ventana Discovery Ultra Immunohistochemistry Autostainer (Roche, Tuscon, AZ, USA) was used for the immunostaining. CD68 (a marker of inflammation; dilution 1:1,000) and CD31 (a marker for endothelial cells; dilution $1: 1,200)$ staining was performed following the optimization of the antibodies for best dilution [11]. The histological sections were digitized using a 3D Histech Scan II Fluorescence/Brightfield Slide Scanner (total magnification $\times 70$ and $0.14-\mu \mathrm{m}$ resolution).

\section{Nanoindentation Test}

Mechanical characterization of the plaque tissue was performed using a Hysitron TI 950 TriboIndenter. The tests were performed using a displacement feedback-controlled mode with a normal stage of $5-\mu \mathrm{m}$ displacement. A conospherical diamond tip, with a radius of curvature of $50 \mu \mathrm{m}$, was used for the experiments. At room temperature, 30 - $\mu$ m-thick sections were indented to a depth of 2,000 nm, which is $6 \%$ of the sample thickness. Sample hydration was maintained by a water-absorbent polymeric foam (OASIS Floral Foam, Auswrap, NSW, Australia) [11].

\section{Computational Fluid Dynamics}

Lumen was segmented using the time of flight sequence in Amira (version 6.0; Thermo Fisher Scientific, Madison, WI, USA). Computational fluid dynamic simulation was performed on ANSYS Workbench (version 19.0; ANSYS, Canonsburg, PA, USA). The 3D lumen geometry was preprocessed using SpaceClaim. Mesh was generated with Tet 4 and Wed6 elements. A mesh size of $0.2 \mathrm{~mm}$ and 10 boundary layers with a growth factor of 1.2 were used for the computational fluid dynamic simulations. Blood was set as a Newtonian fluid with properties of density as $1,050 \mathrm{~kg} / \mathrm{m}^{3}$ and viscosity as $0.00345 \mathrm{~Pa} \cdot \mathrm{s}$. Blood flow was assumed as laminar incompressible and is governed by the Navier-Stokes equation. The time step for the simulations was $0.002 \mathrm{~s}$ running for 2 cardiac cycles; the result from the second cycle was used for computing wall shear stress (WSS). A time-dependent parabolic mass flow rate profile extracted from the 2D ECG-gated PC-MRI was defined at the inlet of the common carotid artery, and the outflow at the internal carotid artery and external carotid artery was defined as the ratio of the inlet profile. WSS values were computed from the analysis.

\section{Structural Analysis}

Different slices corresponding to the selected segments were reconstructed from MRI data of the carotid artery (ImageJ [imagej. nih.gov/ij/]). Mesh size and elastic support stiffness were based on the convergence study. Plane 182 elements with a mesh size of $0.025 \mathrm{~mm}$ with linear elastic isotropic material properties and plane strain behavior were used for the analysis. Elastic support with the stiffness of $0.0005 \mathrm{~N} / \mathrm{mm}^{3}$ was defined on the outer boundary [12]. A uniformly distributed pressure load of $120 \mathrm{~mm}$ $\mathrm{Hg}$ (systolic pressure) was defined on the lumen. Material properties extracted from the experimental values of the nanoindentation 


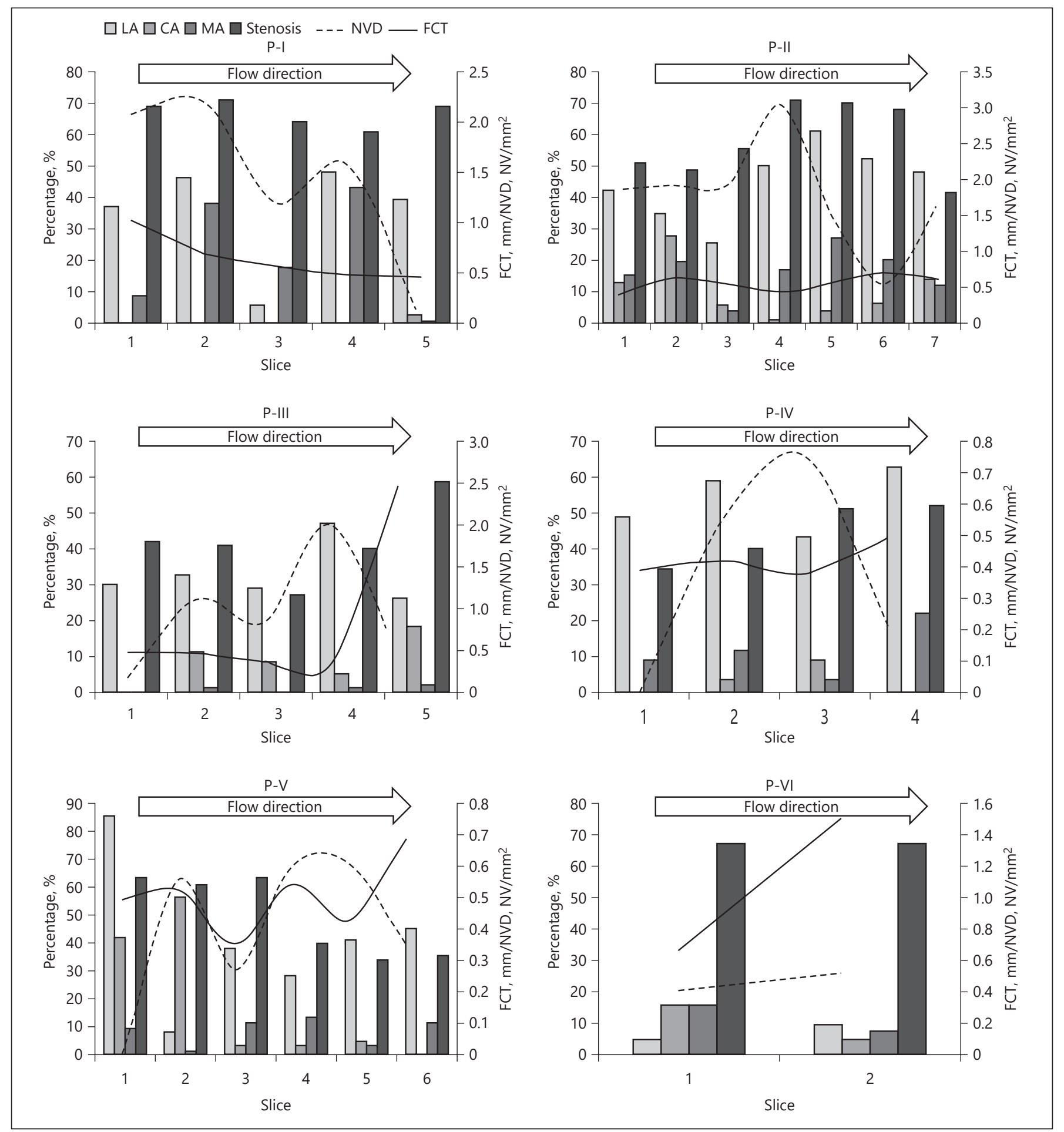

Fig. 1. Quantitative evaluation and correlation of the morphological constituents for 6 patients (P-I to P-VI). The percentage staining for lipid area (LA), calcification area (CA), inflammation (MA), and stenosis is represented on the primary axis, whereas the FCT in $\mathrm{mm}$ and NVD in NV/ $\mathrm{mm}^{2}$ are represented on the secondary axis. The arrow indicates the blood flow direction (upstream to downstream). Individual patient correlations for morphology:
P-II: -ve correlations between $\%$ stenosis and \% CA $\left(r_{\mathrm{s}}=0.964\right.$, $p=0.0004)$ and $\%$ HA and $\%$ LA $\left(r_{s}=0.757, p=0.049\right)$; P-III: FCT and $\%$ stenosis are +vely correlated $\left(r_{\mathrm{s}}=0.9, p=0.037\right)$; P-IV: +ve correlations between $\% \mathrm{MA}$ and $\% \mathrm{LA}\left(r_{\mathrm{s}}=1\right)$, FCT and \% LA $\left(r_{\mathrm{s}}=1\right)$, and FCT and \% MA $\left(r_{\mathrm{s}}=1\right)$; P-V: \% HA and \% stenosis $\left(r_{\mathrm{s}}=0.845, p=0.034\right)$ are +vely correlated. FCT, fibrous cap thickness; NVD, neovessel density. 
test were defined for the respective tissue type. The von Mises stress (VMS) is often used to determine whether a material will yield or fracture when subjected to a complex loading condition. If it is greater than the material's strength, the material would yield or rupture. VMS is a term in solid or material mechanics, which is different from WSS. WSS is a term often used in fluid mechanics, defined as a tangential force imposed by the flowing fluid on the lumen surface.

\section{Data Analysis}

For all the sections analyzed, \% stenosis was calculated according to the European Carotid Surgery Trial (ECST) [13]. The area staining of lipids (LA), calcification (CA), hemorrhage (HA), and macrophages (CD68 staining - MA) was reported as the percentage of the total plaque area calculated from the H\&E stain. Neovascularization was expressed as neovessel density (NVD) neovessels $/ \mathrm{mm}^{2}$. A 2-tailed paired Student $t$ test was used to determine the significance of any differences in morphology and stresses between sections from the upstream and downstream locations of the carotid plaque tissue. Correlations between segmental geometric parameters and stresses along the longitudinal length of each plaque and all sections analyzed were evaluated using nonparametric Spearman's correlations. The calculations were 2-tailed with $p<0.05$ considered statistically significant. Statistical analysis was performed in SPSS 23 (SPSS Inc. IBM).

\section{Results}

\section{Histological Analysis and MRI Segmentation}

Considering all the sections analyzed, it was found that CD68 immunolabeling (inflammation) was positively correlated with lipid $(p=0.026)$ and stenosis $(p=0.019)$. Neovessels (CD31 immunolabeling) were present close to the location of inflammation and lipids. However, no significant correlation was found between the NVD and inflammation. Calcification content was observed to be high in the common carotid region and was correlated with VMS. Quantitative evaluation of the morphology and the corresponding correlations at different sections along the length of each plaque tissue for 6 different patients are represented in Figure 1.

\section{Reduced Moduli and Hardness Values}

There was a notable variation of properties from the same tissue type at different locations on the section and between the sections tested. The sorted data for each tissue type were averaged, and the average reduced modulus values were assigned to the respective tissue in the structural simulations. Reduced modulus values for each tissue type were lipid (median $0.955 \mathrm{MPa}$; range 0.216-1.816 $\mathrm{MPa}$ ), fibrous tissue (median $2.066 \mathrm{MPa}$; range $0.705-$ 12.233 $\mathrm{MPa}$ ), and calcification (median 9.748; $\mathrm{MPa}$; range 2.457-723.103 MPa).

Longitudinal Heterogeneity of the Carotid Plaque Tissue

\section{Wall Shear Stress}

Disturbed flow patterns were observed in the proximity of the bifurcation region and below it. The magnitude of velocity was found to be maximum at the highly stenotic region. The carotid sinus region also represented disturbed velocity streamlines. Figure $2 \mathrm{a}$ and $\mathrm{b}$ represent the low WSS, lumen model, and WSS at different sections along the lumen. Although no significant correlations were found between the average WSS and fibrous cap thickness (FCT), it was observed that average WSS values were high at locations having less FCT and in the highly stenotic ICA region. Figures 3 and 4 represent the variation and correlation of average WSS in relation to morphology for each patient. In particular, the sections with less FCT and calcification area have high average WSS values.

\section{Structural Stress}

Figure $2 \mathrm{c}$ and $\mathrm{d}$ represent the 2D geometries and VMS distribution on different sections in the longitudinal direction. High-stress concentrations were located at the shoulder regions and the regions with a thin fibrous cap covering the lipid core. In addition, the interfaces between calcium and lipid and calcium and fibrous tissue were also subjected to high-stress concentration. Comparing the sections from all the patients, it was found that the change in maximum VMS values for different sections was proportional to the change in percentage of the calcification area $(p=0.007)$. However, the maximum VMS values were negatively correlated with stenosis $(p=$ $0.028)$ and average WSS values $(p=0.034)$. This variation explains that although big chunks of calcification increase the stability of the plaque andhigh-stress concentrations exist in the interface regions between the calcium and the surrounding tissue (fibrous cap and lipid pool). While no significant correlations were observed between VMS and FCT, it was found that VMS values were high at locations where the fibrous cap was thin. Figures 3 and 4 represent the variation and correlation of VMS in relation to morphology for each patient.

Fig. 2. Stress distribution from computational fluid dynamics and structural analysis. Low WSS contour at peak systolic for P-II and $\mathrm{P}-\mathrm{V}$ (a); WSS at different locations along the lumen corresponding to the histological sections (numbered I-VII for P-II and I-VI for $\mathrm{P}-\mathrm{V})$ (b); 2D geometrical models reconstructed based on MRI data for P-II (c); computed von Mises contour plots for different sections along the length of the carotid plaque tissue for P-II (d). WSS, wall shear stress.

(For figure see next page.)

Cerebrovasc Dis 2021;50:510-519 


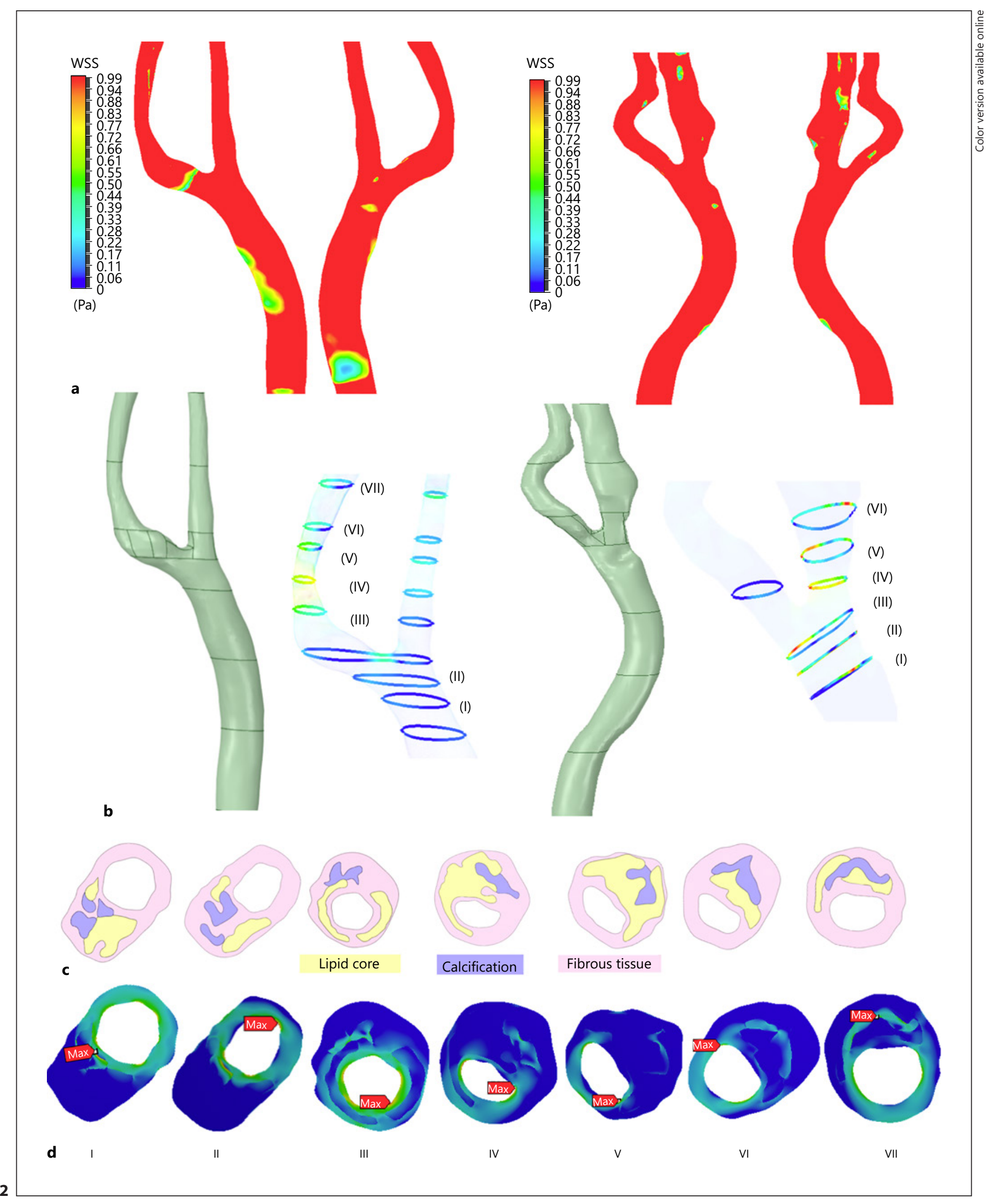



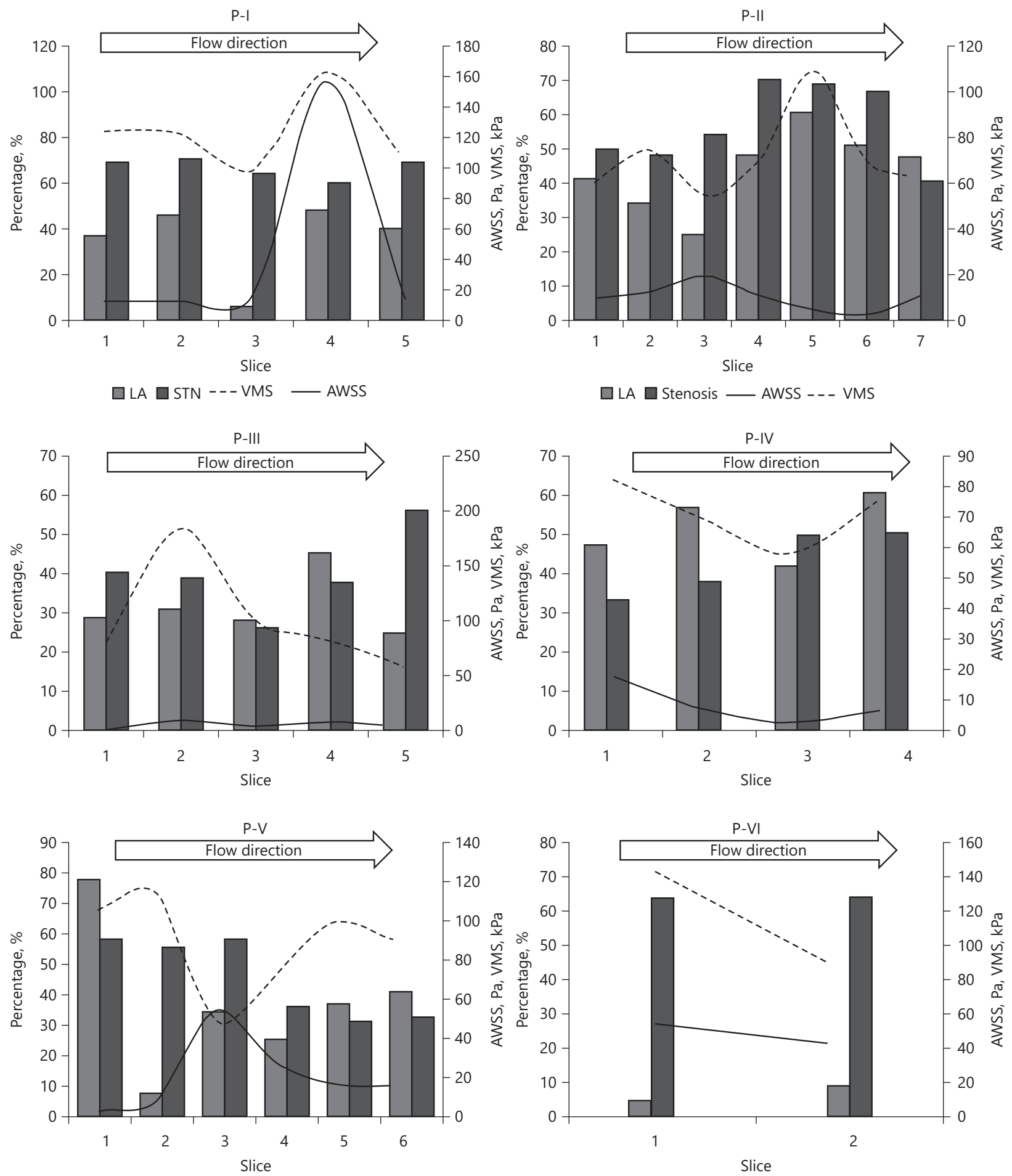

Fig. 3. Quantitative evaluation and correlation between morphology (lipid area and stenosis) and stresses (average WSS and VMS) for 6 patients (P-I to P-VI). The arrow indicates the blood flow direction (upstream to downstream). Individual patient correlations: P-II: average WSS and \% LA are -vely correlated $\left(r_{\mathrm{s}}=0.857, p=0.014\right)$,
VMS and NVD are -vely correlated $\left(r_{s}=0.929, p=0.003\right)$, VMS and average WSS are -vely correlated $\left(r_{\mathrm{s}}=0.786, p=0.036\right)$, and VMS and $\%$ LA are +vely correlated $\left(r_{\mathrm{s}}=0.786, p=0.036\right)$; P-III: average WSS and NVD are +vely correlated $\left(r_{\mathrm{s}}=0.9, p=0.037\right)$. WSS, wall shear stress; VMS, von Mises stress; NVD, neovessel density. 

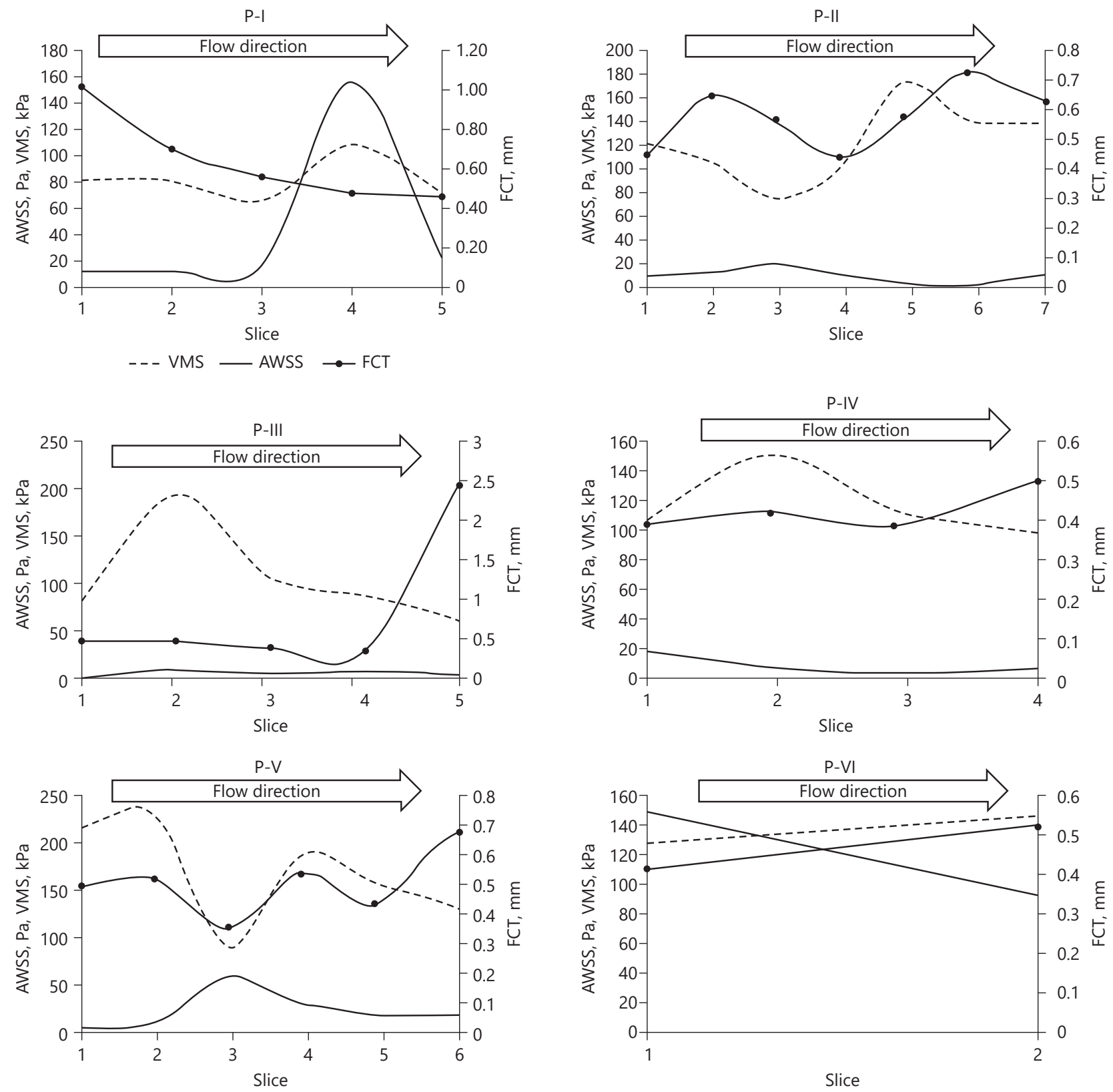

Fig. 4. Quantitative evaluation and correlation between FCT and stresses (average WSS and VMS) for 6 patients (P-I to P-VI). The arrow indicates the blood flow direction (upstream to downstream). Individual patient correlations: P-I: average WSS and FCT are -vely correlated $\left(r_{\mathrm{s}}=0.9, p=0.037\right)$. FCT, fibrous cap thickness; WSS, wall shear stress; VMS, von Mises stress. 


\section{Discussion}

\section{Variation in Plaque Morphology}

Inflammation plays a vital role in the initiation and progression of the disease. The lipid and inflammation followed a similar trend in the longitudinal direction and between all the sections analyzed. This explains the conversion of macrophages to foam cells and subsequently to the necrotic lipid core [14]. It was observed that inflammation and lipid content are high in the stenotic region and in agreement with the literature [15]. In addition, macrophages were present around the lipid area and under the fibrous cap that contribute to the increase in lipid core volume. Previous studies have shown that macrophages in the cap may lead to a softer plaque, resulting in a reduction of stresses, however increasing the strain values [16]. Therefore, cap inflammation combined with a thin fibrous cap may increase the vulnerability of the plaque.

In atherosclerotic lesions, the increase in the percentage of stenosis and inflammatory response reduces oxygen supply resulting in hypoxia, which in turn incites neovascularization [17]. In this study, no correlation was found between inflammation and microvessels density, as reported by Demeure et al. [18].Whereas, Taqueti et al. [19] showed that neovascularization was correlated with inflammation. However, it was interesting to observe that the spatial distribution of neovessels for different sections was associated with the location of inflammation (CD68 immunolabeling). It was also observed that the number of neovessels close to the lumen increased with an increase in cap inflammation. Furthermore, neovessels were found to be in the shoulder region of the plaque.

Individual carotid plaques exhibited heterogeneity in composition and morphology along the length of the plaque. The differences in plaque morphology and composition at different locations along the length of the plaque represent different stages of the plaque development. Plaque heterogeneity combined with hemodynamic characteristics and pathophysiology could result in further plaque progression or regression. Therefore, evaluating plaque morphology and understanding the heterogeneity of an individual plaque could provide insights into the risk stratification.

\section{Variation in Modulus Values}

The reduced modulus values varied between the same tissue type at different locations of the same section and between sections. The properties solely depended on the tissue type, its structure, and neighboring tissue. The reduced modulus values of the fibrous tissue were influ- enced by the compositional changes and were high at regions with structured collagen in comparison with the unstructured collagen as reported by the previous literature [9]. The structured collagen has a complex orientation of the fibers that increase the strength of the tissue. The mean reduced modulus values obtained for different tissue types were consistent with the higher range of values obtained in the literature [20].

\section{Variation in Stresses}

WSS values varied along the length of the plaque with higher values at the midplaque region and low values at upstream and downstream regions. Low WSS regions indicate the locations vulnerable to the initiation of the plaque, and high WSS regions indicate the direction of the plaque growth. The average WSS values are high at locations of the thin fibrous cap and calcification. Higher values of WSS incite plaque progression and might result in the rupture of the thin fibrous cap leading to an acute event. Therefore, FCT is an important parameter in analyzing the plaque vulnerability $[21,22]$. Furthermore, in comparison with the region opposite to the plaque, low WSS was found on the lumen covering the plaque. This explains the influence of low WSS on the plaque initiation locations. Based on the WSS distribution and percentage of stenosis along the length of the plaque, it is evident that plaque may progress downstream. Previous studies have also shown that plaques progress longitudinally, along blood flow 2.4 times faster, than they thicken [23].

Dynamic interaction between patient-specific risk factors, hemodynamic factors, and biomechanical environment instigates endothelial dysfunction thereby initiating atherosclerosis. The arteries exposed to WSS variations influenced by flow characteristics contribute to the initiation and progression of the plaque. Therefore, it must be noted that hemodynamic factors are clearly important in atherosclerosis, and drugs that affect hemodynamic factors $[24,25]$ appear to affect atherosclerosis. WSS values combined with plaque morphology could help us in identifying the risk of rupture and providing patient-specific diagnosis and treatment strategies.

Maximum VMS values were found to act in the shoulder regions or at the locations of the thin fibrous cap covering the lipid core. High-stress concentrations were also found in the interface regions of different tissue types, especially around the calcification and the surrounding tissue, thereby increasing the plaque vulnerability [26]. The geometry of the calcification also affects plaque vulnerability [27]. If observed along the length of the plaque, maximum VMS values varied with the lipid and calcification area. In addi- 
tion, the peak stresses and neovessels were found to be in the shoulder regions. In summary, although there were variations in the stress values along the longitudinal direction, the carotid plaque tissue revealed no significant difference in WSS and maximum VMS between the upstream and downstream locations of the carotid plaque tissue.

Plaque rupture is influenced by the structural stresses (VMS) developed due to the arterial remodeling of the vessel wall, in response to endothelial dysfunction influenced by the biomechanical environment of the vasculature. If the structural stresses of the fibrous cap exceed the strength of the material, the plaque ruptures. In addition, stress concentration often happens at the material interface which could be a favorite site for initiation of rupture. Thus, plaque morphology, heterogeneity, and structural stresses play a very important role in determining the vulnerability of the plaque.

\section{Study Limitations}

This study provides valuable information about tissue heterogeneity and mechanobiological correlations. However, there are inherent limitations in this study. First, the tissue sample was slit longitudinally during carotid endarterectomy. Therefore, the shape of the histology image was out of its original shape, which might cause an error while registering the histology and MRI data. Second, the resolution of MRI was another limitation of this study. The slice thickness between MRI and histology was different, which could affect the coregistration between them. Third, though the $30-\mu \mathrm{m}$ slices were obtained via cryosectioning, there was a difference in the thickness of the samples. This variation is due to the components present, especially the calcification. Despite indenting the samples to a thickness $<6 \%$ of the total thickness, the values have been influenced by the substrate effects at locations having gaps in the sample due to loss of calcification or lipids during sample preparation and unstructured tissue as visible from histology. Fourth, 2D reconstructed models were used for structural analysis. Lastly, only 6 samples were included in the study. Future studies might use 3D reconstructed geometries for the fluid-structure interaction simulations with patientspecific boundary conditions in combination with structural and mechanical characterization.

\section{Conclusion}

In this study, longitudinal variations in morphology, mechanical properties, and stresses were analyzed. Significant variations and correlations suggest that arterial remod- eling is a dynamic interaction between mechanical forces and plaque progression resulting in longitudinal heterogeneity, influenced by patient-specific risk factors, hemodynamic factors, and biomechanical environment. The results obtained in this study are a step forward towards enhancing the understanding of the mechanobiological environment and tissue heterogeneity in the longitudinal direction.

\section{Acknowledgements}

The authors would like to thank the team at PA Hospital for identifying potential research participants and providing facilities for a low-energy X-ray, especially Gillian Jagger. The authors would also like to acknowledge the technical support of QUT's Institute of Health and Biomedical Innovation and Central Analytical Research Facility.

\section{Statement of Ethics}

This study was approved by the Human Research Ethics Committee at the Princess Alexandra Hospital (PAH) in Brisbane, Australia, and QUT's Office of Research Ethics and Integrity (HREC/17/ QPAH/181). All procedures performed in the studies were in accordance with the ethical standards of the institutional and/or a national research committee. Informed consent and consent to publish were obtained from the participants included in the study.

\section{Conflict of Interest Statement}

The authors declare that they have no conflicts of interest.

\section{Funding Sources}

This work was supported by the Australian Research Council (ARC) (DP200103492 and DP200101970), the PA Research Foundation (PARF), and the National Nature Science Foundation of China (11972118, 11772093, and 61821002).

\section{Author Contributions}

The work was designed by P.P., P.Y., and Z.L. Histological analysis was performed by P.P. and T.Y. Nanoindentation experiments were performed by P.P. Mechanical simulations were performed by P.P., J.M., and J.W. T.M. and T.L. provided their expertise in MRI and vascular mechanics. P.P. wrote the manuscript text and analyzed results. All authors reviewed the manuscript. 


\section{References}

1 Morbiducci U, Kok AM, Kwak BR, Stone PH, Steinman DA, Wentzel JJ. Atherosclerosis at arterial bifurcations: evidence for the role of haemodynamics and geometry. Thromb Haemost. 2016;115(3):484-92.

2 Benjamin EJ, Virani SS, Callaway CW, Chamberlain AM, Chang AR, Cheng S, et al. Heart disease and stroke statistics-2018 update: a report from the American Heart Association. Circulation. 2018;137(12):e67-e492.

3 Bogiatzi C, Hackam DG, McLeod AI, Spence JD. Secular trends in ischemic stroke subtypes and stroke risk factors. Stroke. 2014;45(11): 3208-13.

4 Chai JT, Biasiolli L, Li L, Alkhalil M, Galassi F, Darby C, et al. Quantification of lipid-rich core in carotid atherosclerosis using magnetic resonance T 2 mapping: relation to clinical presentation. JACC Cardiovascular Imaging. 2017 Jul;10(7):747-56.

5 Teng Z, He J, Degnan AJ, Chen S, Sadat U, Bahaei NS, et al. Critical mechanical conditions around neovessels in carotid atherosclerotic plaque may promote intraplaque hemorrhage. Atherosclerosis. 2012;223(2):321-6.

6 Li ZY, Howarth S, Trivedi RA, U-King-Im JM, Graves MJ, Brown A, et al. Stress analysis of carotid plaque rupture based on in vivo high resolution MRI. J Biomech. 2006;39(14): 2611-22.

7 Li ZY, Howarth SP, Tang T, Graves MJ, UKing-Im J, Trivedi RA, et al. Structural analysis and magnetic resonance imaging predict plaque vulnerability: a study comparing symptomatic and asymptomatic individuals. J Vasc Surg. 2007;45(4):768-75.

8 Walsh MT, Cunnane EM, Mulvihill JJ, Akyildiz AC, Gijsen FJ, Holzapfel GA. Uniaxial tensile testing approaches for characterisation of atherosclerotic plaques. J Biomech. 2014; 47(4):793-804

9 Chai CK, Akyildiz AC, Speelman L, Gijsen FJ, Oomens CW, van Sambeek MR, et al. Local axial compressive mechanical properties of human carotid atherosclerotic plaques-characterisation by indentation test and inverse finite element analysis. J Biomech. 2013; 46(10):1759-66.
10 Ebenstein DM, Coughlin D, Chapman J, Li C, Pruitt LA. Nanomechanical properties of calcification, fibrous tissue, and hematoma from atherosclerotic plaques. J Biomed Mater Res A. 2009;91(4):1028-37.

11 Paritala PK, Yarlagadda T, Benitez Mendieta J, Wang J, Gu Y, Li Z, et al. Characterization of the atherosclerotic plaque tissue. Aml. 2020;11(5):20051507. Article number: 20051507 1-7.

12 Paritala PK, Yarlagadda PKDV, Wang J, Gu Y, Li Z. Numerical investigation of atherosclerotic plaque rupture using optical coherence tomography imaging and XFEM. Eng Fract Mech. 2018;204:531-41.

13 Staikov IN, Arnold M, Mattle HP, Remonda L, Sturzenegger M, Baumgartner RW, et al. Comparison of the ECST, CC, and NASCET grading methods and ultrasound for assessing carotid stenosis. European carotid surgery trial. North American symptomatic carotid endarterectomy trial. J Neurol. 2000;247(9):681-6.

14 Lusis AJ. Atherosclerosis. Nature. 2000; 407(6801):233-41.

15 Grønholdt MLM, Nordestgaard BG, Bentzon J, Wiebe BM, Zhou J, Falk E, et al. Macrophages are associated with lipid-rich carotid artery plaques, echolucency on B-mode imaging, and elevated plasma lipid levels. J Vasc Surg. 2002;35(1):137-45.

16 Tang D, Yang C, Huang S, Mani V, Zheng J, Woodard PK, et al. Cap inflammation leads to higher plaque cap strain and lower cap stress: an MRI-PET/CT-based FSI modeling approach. J Biomech. 2017;50:121-9.

17 Sluimer JC, Daemen MJ. Novel concepts in atherogenesis: angiogenesis and hypoxia in atherosclerosis. J Pathol. 2009;218(1):7-29.

18 Demeure F, Bouzin C, Roelants V, Bol A, Verhelst R, Astarci P, et al. Head-to-head comparison of inflammation and neovascularization in human carotid plaquesCLINICAL PERSPECTIVE: implications for the imaging of vulnerable plaques. Circ Cardiovasc Imaging. 2017;10(5):e005846.
19 Taqueti VR, Di Carli MF, Jerosch-Herold M, Sukhova GK, Murthy VL, Folco EJ, et al. Increased Microvascularization and vessel permeability associate with active inflammation in human atheromata. Circ Cardiovasc Imaging. 2014;7(6):920-9.

20 Ebenstein DM, Chapman JM, Li C, Saloner D, Rapp J, Pruitt LA. Assessing structure-property relations of diseased tissues using nanoindentation and FTIR. MRS Online Proc Libr Arch. 2001;711.

21 Zahnd G, Schrauwen J, Karanasos A, Regar E, Niessen W, van Walsum T, et al. Fusion of fibrous cap thickness and wall shear stress to assess plaque vulnerability in coronary arteries: a pilot study. Int J Comput Assist Radiol Surg. 2016;11(10):1779-90.

22 Saba L, Potters F, Van Der Lugt A, Mallarini G. Imaging of the fibrous cap in atherosclerotic carotid plaque. Cardiovasc Intervent $\mathrm{Ra}$ diol. 2010;33(4):681-9.

23 Barnett PA, Spence JD, Manuck SB, Jennings JR. Psychological stress and the progression of carotid artery disease. J Hypertens. 1997; 15(1):49-55.

24 Spence JD, Perkins DJ, Kline RL, Haust MD. Aortic atherosclerosis in hypertensive rabbits treated with anti-hypertensive agents having different effects on arterial flow disturbances. 1. Extent of surface involvement. Paroi Arterielle. 1981;7(4):177-87.

25 Spence JD, Pesout AB, Melmon KL. Effects of antihypertensive drugs on blood velocity in rhesus monkeys. Stroke. 1977;8(5):58994.

26 Hoshino T, Chow LA, Hsu JJ, Perlowski AA, Abedin M, Tobis J, et al. Mechanical stress analysis of a rigid inclusion in distensible material: a model of atherosclerotic calcification and plaque vulnerability. Am J Physiol Heart Circ Physiol. 2009;297(2):H802-10.

27 Buffinton CM, Ebenstein DM. Effect of calcification modulus and geometry on stress in models of calcified atherosclerotic plaque. Cardiovasc Eng Tech. 2014;5(3):244-60. 